\title{
Stage IB Lung Cancer AJCC v7
}

National Cancer Institute

\section{Source}

National Cancer Institute. Stage IB Lung Cancer A/CC v7. NCI Thesaurus. Code C5643.

Stage IB includes: T2a, N0, M0. T2a: Lung cancer with a tumor size more than $3 \mathrm{~cm}$ but $5 \mathrm{~cm}$ or less in greatest dimension. N0: No regional lymph metastasis. M0: No distant metastasis. (AJCC 7th ed.) 